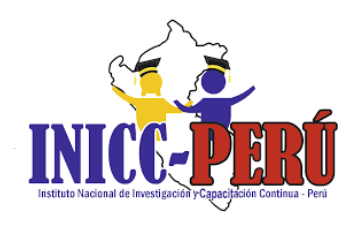

\title{
Estrés académico en estudiantes de nuevo ingreso de Licenciatura en Psicología de la Universidad de Holguín, Cuba
}

\author{
Academic stress in new students of bachelor's Degree in Psychology \\ at the University of Holguín, Cuba
}

MAIDÉ PÉREZ AGUILERA ${ }^{1}$

https://orcid.org/0000-0002-6754-9642

\section{YUDISLEIDYS HERNÁNDEZ ROJAS ${ }^{2}$}

https://orcid.org/0000-0002-8646-8880

\section{ERNESTO SANTIESTEBAN LEYVA ${ }^{3}$}

https://orcid.org/0000-0001-7028-7172

\begin{abstract}
RESUMEN: Recientemente se han incrementado los problemas de salud física y emocional en la población asociados al estrés. Según diversos estudios de la Organización Mundial de la Salud (OMS), una cantidad cada vez más significativa de personas en todo el mundo sufren de estrés. Con la presente investigación se pretendió analizar cuáles son los indicadores del estrés académico que se constatan en un grupo de primer año de la carrera Licenciatura en Psicología de la Universidad de Holguín, dadas una serie de insuficiencias que se presentaron en estos en forma de sintomatología clínica, que estaban entorpeciendo el desarrollo sano de la personalidad. La investigación permitió conocer la presencia de indicadores de estrés académico moderado y valorar críticamente sus estrategias de afrontamiento.
\end{abstract}

Palabras clave: estudiantes universitarios; estrés; estrés académico; estresores; síntomas; estrategias de afrontamiento.

ABSTRACT: Recently, physical and emotional health problems associated with stress have increased in the population. According to various studies by the World Health Organization (WHO), an increasingly significant number of people around the world suffer from stress. This research aimed to analyze which are the indicators of academic stress that emerge in a group of first year of the Bachelor's degree in Psychology at the University of Holguín, given a series of deficiencies that appeared in these in the form of clinical symptoms, which were hindering the healthy development of the personality. The research allowed to know the presence of indicators of moderate academic stress and to critically assess their coping strategies. 
Keywords: College Students; Stress; Academic Stress; Stressors; Symptoms; Coping Strategies.

\section{INTRODUCCIÓN}

El desarrollo integral de la personalidad del estudiante cubano en cualquiera de sus ámbitos de aprendizaje, constituye el principal objetivo de nuestro sistema de educación. El tránsito hacia al contexto universitario exige de éstos desarrollar una serie de habilidades, actitudes y valores que les permitan una adaptación activa a las nuevas exigencias, presupone establecer nuevos tipos de relaciones entre iguales, de pareja y también implica nuevas oportunidades de libertad y de experiencias, ya mucho más independientes del ámbito familiar.

En esta nueva etapa en la vida del joven, su desarrollo psicológico y estilo de vida deberá reorganizarse para corresponderse al aumento del rigor del estudio, la multiplicidad de tareas docentes, encarar nuevas relaciones sociales, etcétera. En esta situación, resulta fundamental que los mismos estén preparados para evitar cualquier conducta riesgosa y establecer un equilibrio entre las nuevas exigencias y las potencialidades para su desarrollo.

Recientemente se han incrementado los problemas de salud física y emocional en la población asociados al estrés. Según diversos estudios de la Organización Mundial de la Salud (OMS), una cantidad cada vez más significativa de personas en todo el mundo sufren de estrés. En el mundo Caldera, Pulido y Martínez (2007), "uno de cada cuatro individuos sufre de algún problema grave de estrés y en las ciudades, se estima que el 50 por ciento de las personas tienen algún problema de salud mental de este tipo" (p. 78). Esta es una de las patologías que se presentaron mayor frecuencia en la actualidad.

El estrés es el comienzo de una serie de enfermedades y, aunque no es la causa directa de las mismas, con frecuencia contribuye a su desarrollo.

Fisher (1986) considera que la entrada en la Universidad, con los cambios que esto supone, representa un conjunto de situaciones altamente estresantes debido a que el individuo puede experimentar, aunque en ocasiones transitoriamente, una falta de control sobre el nuevo ambiente, potencialmente generador de estrés y, en último término, potencial generador con otros factores del fracaso académico universitario.

En una exhaustiva revisión bibliográfica sobre el la problemática del estrés académico en la universidad, pudimos constatar que en nuestro país las investigaciones sobre el tema son más limitadas con relación a países europeos y de Latinoamérica, donde se considera, es un fenómeno generalizado en los primeros años de estudios universitarios.

Un estudio exploratorio realizado en el año 2002 en Ciudad de La Habana, para determinar algunas características del estrés, reflejó como la mayoría de los estudiantes, el 69,2\%, es vulnerable al estrés citado en Díaz, Y (2010). Román, Ortiz y Hernández (2008), constataron la elevada vulnerabilidad al estrés en una muestra de estudiantes de primer año de la carrera de Medicina en la Escuela Latinoamericana de Medicina (ELAM).

El uso del término estrés se ha popularizado sin que la mayoría de las personas tengan claro en qué consiste el mismo. Al revisar la amplia bibliografía sobre el tema, se encuentran 
multitud de definiciones, se prefiere tomar en consideración lo señalado por Zaldívar (2011) quien expresa que:

Se analiza el estrés académico como sinónimo de distrés, obviando la dimensión de estrés como una variante de este. En muchas investigaciones se observa la limitación de que se le atribuye un papel negativo al estrés como elemento perturbador, excluyendo al estrés como la necesaria activación basal para la adaptación a la demanda ambiental, aun cuando la experiencia del estrés puede convertirse en un importante factor de desarrollo y crecimiento personal, así como una herramienta de gran utilidad para el logro de un funcionamiento armónico y del mantenimiento de la salud (p. 45).

A decir este autor se vive inmerso en el estrés, porque los sujetos se encuentran en un proceso constante de adaptación al mundo que les rodea, y a la vez del propio desarrollo personal, lo que provoca realizar constantes esfuerzos cognitivos y conductuales para mejorar las situaciones en que se presentan.

Los estudios más recientes sobre procesos de estrés, han puesto en consideración sus aspectos positivos y el papel que este puede desempeñar como factor de desarrollo y crecimiento personal, cuando se logra un eficiente manejo del mismo, y que los estudiantes que tienen un estilo de vida estructurado hacia la consecución de sus metas escolares y presentan un estilo centrado en la solución de problemas, son menos vulnerables al estrés.

Uno de los autores más que más resultados ha logrado en los estudios sobre estrés académico es Barraza, A (2006) quien defiende la siguiente idea: el estrés académico es aquel que padecen los alumnos de educación media superior y superior y que tiene como fuente exclusiva a estresores relacionados con las actividades a desarrollar en el ámbito escolar. Esta definición se circunscribe al estrés que padecen los alumnos a consecuencia de las exigencias endógenas o exógenas que impactan su desempeño en el ámbito escolar.

Según este autor, el concepto se puede ilustrar de la siguiente manera: el alumno se ve sometido, en contextos escolares, a una serie de demandas que, tras ser valoradas como estresores, provocan un desequilibrio sistémico (situación estresante); este se manifiesta en una serie de síntomas (indicadores del desequilibrio) que obliga al alumno a realizar acciones de afrontamiento. Esta forma de conceptualizar al estrés académico permite reconocer tres componentes sistémico-procesuales: estímulos estresores, síntomas (indicadores del desequilibrio sistémico) y estrategias de afrontamiento.

Autores como Polo, A., Hernández, J: M., y Poza, C. (2009), precisan como causantes del estrés académico: realización de exámenes, exposición de trabajos, sobrecarga académica, falta de tiempo para cumplir con las actividades, competitividad entre compañeros, entre otros. Por su parte Barraza (2007) agrega: sobrecarga de tareas, mantener un buen promedio, falta de incentivos, problemas personales entre compañeros, exceso de responsabilidad, etc.

El estrés académico en el mundo es considerado, una problemática a la que se está prestando una atención creciente. Con relación a lo anterior se observa una ausencia de mecanismos y procesos de ajuste institucional (al menos de forma intencionada) en la universidad, de mecanismos y herramientas psicológicas de modo que provean de recursos no solo al individuo sino también a la institución, para el manejo del estrés académico desde una 
perspectiva educativa global que estimule el desarrollo de la personalidad del individuo de modo que sea poco vulnerable al estrés. No se aprecia una reorientación del funcionamiento del proceso docente educativo y a la vida de la comunidad universitaria con vistas a minimizar los eventos estresores, así como el desarrollo psicopedagógico del estudiante.

La experiencia como profesora ha posibilitado apreciar que cuando el estudiante de psicología del curso por encuentros ${ }^{4}$, se enfrenta a su nueva realidad como estudiante universitario, con las exigencias y habilidades que debe desarrollar para enfrentar las tareas, comienza a manifestar una serie de conductas que van desde: reacciones defensivas, hasta una serie de sintomatologías clínicas, tanto físicas como psicológicas, que entorpecen el proceso de enseñanza aprendizaje y frenan el desarrollo de la personalidad sana.

La carga docente exige del estudiante del curso por encuentros, permanecer en clase dos fines de semana al mes con dos sesiones generalmente de seis horas clase. Importante aspecto es para los mismos, la asistencia a clases, y trasladarse de su lugar de residencia a la sede universitaria puede resultar, sobre todo para aquellos que residen lejos de la misma, un esfuerzo significativo.

Además de tiempo dedicado a recibir docencia directa, al estudiante se le exige integrarse a un grupo de actividades de extensión universitaria, a las que dedica un tiempo variable según su naturaleza.

Cada una de las materias, al margen de variaciones individuales que hacen imprevisible este aspecto, aunque la modalidad de curso por encuentros presupone que el estudiante dedique en el período que media entre un encuentro y otros un mínimo de dos horas por asignatura a estudiar de modo independiente, ya sea individualmente o por equipos, el contenido orientado en cada encuentro.

El estudiante del curso por encuentro desarrolla su docencia en su doble condición de trabajador- estudiante. En la organización del tiempo dedicado a sus deberes docentes atraviesan de manera decisiva las exigencias que le imponen el desempeño de su profesión y las exigencias de su vida familiar y privada. La dedicación a sus deberes docentes estará siempre en dependencia de la prioridad que en cada caso le otorgue al cumplimiento de estos deberes en relación con el resto de las exigencias profesionales, familiares y personales.

Lo anterior nos brinda una idea de las exigencias que, en dedicación de tiempo y exigencias de índole académica, recaen sobre el estudiante de primer año de la carrera. En ese sentido, los estudiantes de psicología pueden verse afectados debido a que aún no cuentan con todos los recursos personales y sociales necesarios para hacer frente a demandas excesivas de estrés, pero a su vez constituye una potencialidad, que puedan reconocer por la formación que reciben el riesgo a que están expuestos, por la posibilidad que los saberes que la carrera les brinda, para identificar sus limitaciones personales y emocionales para afrontar el estrés académico.

Todo lo anterior expuesto cobra sentido cuando apreciamos que, en un estudio realizado a estudiantes del primer año de la Carrera de Licenciatura en Psicología de la Universidad de

4. Curso por encuentros: modalidad de estudios que se caracteriza por encuentros presenciales en la universidad con frecuencia quincenal donde el estudiante recibe orientaciones para desarrollar sus estudios por su cuenta siendo evaluado en el encuentro siguiente. (Nota de la Autora). 
Holguín, mostró insuficiencias relacionadas con el manejo de la vivencia del estrés académico, entre las que se destacan:

Los estresores que se identifican: La sobrecarga de tareas, la dificultad para organizar el tiempo, la dificultad en la solución de problemas y la sobrecarga académica.

La sintomatología clínica que predominan: Presencia de sentimientos de preocupación y nerviosismo, ansiedad, problemas de concentración, cefalea, trastornos del sueño, astenia para realizar las labores escolares.

Las estrategias de afrontamiento a las cuales recurrieron con más frecuencia fueron: la habilidad asertiva, la elaboración de un plan y ejecución de sus tareas, la ventilación y las confidencias y distraerse viendo televisión, pero éstas no están siendo del todo efectivas para el afrontamiento al estrés académico, ya que no cumplen con su función que sería restablecer el equilibrio sistémico o eliminar síntomas.

Es necesario destacar que la mirada pedagógica sobre la problemática, se centra no tanto en las dificultades de las personas, sino en identificar un amplio abanico de experiencias enriquecedoras, enmarcadas en las posibilidades de desarrollo y de aprendizaje, a partir de la vivencia del estrés académico. Es por ello, que se propone como objetivo: Analizar cuáles son los indicadores del estrés académico que emerge en un grupo de primer año de la carrera Licenciatura en Psicología de la Universidad de Holguín.

\section{METODOLOGÍA}

Los métodos y técnicas utilizados para los fines de la investigación fueron:

Observación: para constatar la presencia o no de sintomatología clínica.

Entrevistas: para profundizar en los estresores percibidos o no, así como usan las estrategias de afrontamiento ante el estrés académico.

Estudio de casos: para constatar como los recursos personológicos de un sujeto pueden potenciar o no la emergencia del estrés académico.

Inventario del SISCO sobre el estrés académico: para evaluar, estresores, síntomas y estrategias de afrontamiento.

La Población la constituyen 314 estudiantes de la carrera Psicología en la Universidad de Holguín, y para la Muestra se escogen intencionalmente, 30 estudiantes de nuevo ingreso con manifestaciones del estrés académico.

\section{RESULTADOS Y DISCUSIÓN}

Con el diagnóstico realizado se pudo constatar en los estudiantes de primer año de la carrera de Licenciatura en Psicología de la Universidad de Holguín, la emergencia de indicadores de estrés académico. Se observan la presencia de sentimientos de preocupación o nerviosismo en la totalidad del grupo de estudiantes lo que sugiere la probabilidad de estrés académico en los mismos. 
En análisis detallado de las técnicas aplicadas se pudo comprobar que los estresores académicos que refieren con mayor frecuencia los alumnos son: dificultad en la organización del tiempo, este se presenta en la totalidad y con una mayor intensidad, al indagar como organizan su tiempo así como se planifican para cumplir las demandas académicas o cuales estrategias consideran más efectivas, pudimos constatar que la mayoría de ellos no tiene un plan estructurado que organice su tiempo, más bien usan estrategias en forma de ensayo y error que dan al traste con la emergencia del estrés académico.

Se observa una tendencia a referir sobrecarga académica, de estudio, de tareas o de trabajo escolares, este estresor es a mi entender más percibido que real y lo relaciono con los problemas de organización temporal referidos anteriormente, lo cual posibilita que se vivencie por su parte esta sobrecarga al no saber discriminar entre lo importante de lo urgente y así organizar su tiempo de forma tal que favorezca una actividad de estudio eficiente y libre de estrés.

Se aprecia además dificultad para solucionar problemas, al igual que la realización de un examen de tipo oral, la forma de evaluación de los profesores como trabajos de investigación, búsquedas en Internet, etc., los cuales también se relacionan con la dificultad para organizar su tiempo, así como la percepción de falta de capacidad para hacer bien los trabajos que piden los profesores, y dificultad para comunicarse con los mismos, y que impresiona este asociado a la dificultad que tienen estos estudiantes de primer año para apropiarse del lenguaje de la ciencia, pues refieren dificultad en las asignaturas propias de la ciencias psicológicas, lo cual se convierte en una barrera en la comunicación.

Es válido señalar como la variable sobrecarga de tareas, se ve afectada en un porciento mayor en mujeres que en hombres, aquí confluyen varios aspectos que dan al traste con ese resultado y es que en la carrera de psicología prevalecen las féminas a los hombres y unido a esto, la mujer en su rol como estudiante universitaria; tiene que ser ama de casa, esposa, y cumplir con todo lo asignado socioculturalmente a su condición de mujer, lo cual posibilita la emergencia de la vivencia estrés académico con más frecuencia en estas, por lo cual se hace necesario profundizar en estudios posteriores al estrés académico desde la categoría género. La sintomatología más frecuente que manifiestan los universitarios son: trastornos en el sueño (insomnio mayor necesidad de dormir), sudor en las palmas de las manos, problemas digestivos, migraña o dolor de cabeza, dolor en el cuello o en la parte baja de la espalda, inquietud y nerviosismo, preocupación excesiva, dificultad para concentrarse, sensación de inseguridad, irritabilidad, y en menor medida, competitividad.

Al analizar las estrategias de afrontamiento a la que recurren de forma general los estudiantes universitarios; se aprecia que los niveles de estrés académico son altos y que las estrategias de afrontamiento que más sobresalen; no están siendo efectivas para la gestión del estrés académico por su parte, entre las cuales puntean: habilidad asertiva defender nuestras preferencias, ideas o sentimientos sin dañar a otros, escuchar música o distraerse viendo televisión, concentrarse en resolver la situación que les preocupa, búsqueda de información sobre la situación que les preocupa, solicitar el apoyo de familiares o de amigos y mantener el control sobre las emociones para que no les afecte lo que les estresa, este al ser una de las más referidas como casi siempre, denota como estas estrategias no están siendo efectivas, pues predomina más la estrategia centrada en la emoción que en la de la solución de problemas. 
Todo lo anterior descrito, coincide parcialmente con los resultados obtenidos en la Universidad Central "Marta Abreu" de Las Villas donde se comenzó el proceso de validación del Inventario SISCO del estrés académico de (Barraza, 2007). Lo cual nos lleva a reflexionar que el problema del estrés académico es más real de lo que aparenta, ya que mediante una investigación instrumental con una muestra de 343 estudiantes de primer y tercer año de las diferentes carreras de dicha institución (Jiménez Jorge y Nieves (2013). Entre los principales resultados de la investigación referenciada, se describió la presencia de estrés académico profundo, siendo mayor en las mujeres. Las situaciones estresantes que sobresalen son la sobrecarga de tareas extra clases y trabajos independientes, las evaluaciones y el tipo de trabajo que exigen los profesores, la participación en clases y el tiempo limitado para la realización de estos trabajos. Entre los síntomas que experimentan los estudiantes ante esta situación, predominan (...), la inquietud y los problemas de concentración; (...) los dolores de cabeza y migrañas, la somnolencia o mayor necesidad de dormir; (...), el desgano para realizar las labores escolares. Las estrategias de afrontamiento más frecuentes fueron: la habilidad asertiva, la elaboración de un plan y ejecución de sus tareas, la ventilación y las confidencias.

En el diagnóstico realizado, emergió coincidiendo con Fernández (2007), como ciertos contenidos de la personalidad pueden propiciar o no un afrontamiento activo y efectivo al estrés académico, como pueden ser la motivación, la relación madurez-inmadurez como indicador de regulación personológicos, la capacidad de autorregulación del comportamiento de forma consciente o formaciones motivacionales más complejas como la autovaloración, y la concepción del mundo, etc. La autorregulación del comportamiento de forma consciente en el afrontamiento ante el estrés, constituye el indicador por excelencia de la personalidad saludable. Pues resulta imprescindible que se produzca en el sujeto la formación de mecanismos y contenidos psicológicos que le permitan, como tendencia de su actuación, organizar de manera estable, consciente y voluntaria su comportamiento.

Todas esas tendencias anteriormente descritas, apuntan hacia un diagnóstico favorable en pos de un desenvolvimiento sano de la personalidad y una gestión adecuada de su estrés, el cual, de no ser contenido a tiempo, podría poner en peligro el mantenimiento de su salud, tanto física como psicológica.

Llama la atención como los estudiantes más estresados son los que mejores resultados académicos obtienen, así como los que más se esfuerzan por cumplir las tareas escolares y los de mejor asistencia a clases, lo cual pudiera estar hablando de potencialidades para el desarrollo que de ser identificadas a tiempo por parte de los profesores u orientadores educativos, ese estrés académico pudiera, bien manejado convertirse en un facilitador para el desarrollo de una personalidad sana en el estudiante universitario.

A partir de estos resultados se infiere que el estrés académico que emerge en algunos estudiantes en sus primeros años de la carrera, pudiera ser transitoria y convertida en un impulso para el desarrollo, si se identifica a tiempo y se trabaja en la estimulación de sus potencialidades. Esta inferencia está basada en la identificación de estresores académicos que guardan relación con problemas de adaptación y transición dentro del sistema educativo, que no estaban presentes en enseñanzas precedentes y que se perciben por estos como desbordantes. 


\section{CONCLUSIONES}

Con el diagnóstico realizado se pudo constatar en los estudiantes de primer año de la carrera de Licenciatura en Psicología de la Universidad de Holguín, la emergencia de indicadores de estrés académico:

- Los estresores que se identifican: La sobrecarga de tareas, la dificultad para organizar el tiempo, la dificultad en la solución de problemas y la sobrecarga académica.

- La sintomatología clínica que predominan: Presencia de sentimientos de preocupación y nerviosismo, ansiedad, problemas de concentración, cefalea, trastornos del sueño, astenia para realizar las labores escolares.

- Las estrategias de afrontamiento a las cuales recurrieron con más frecuencia fueron: la habilidad asertiva, la elaboración de un plan y ejecución de sus tareas, la ventilación y las confidencias y distraerse viendo televisión, pero llama la atención que éstas no están siendo del todo efectivas para el afrontamiento al estrés académico, ya que no cumplen con su función que sería restablecer el equilibrio sistémico o eliminar síntomas.

Se constató, además, como ciertos contenidos de la personalidad pueden propiciar o no un afrontamiento activo y efectivo al estrés académico, por lo que se infiere que la vivencia del estrés académico que emerge en algunos estudiantes en sus primeros años de la carrera, pudiera ser transitoria y convertida en un impulso para el desarrollo, si se identifica a tiempo y se trabaja en la estimulación de las potencialidades.

\section{REFERENCIAS}

Alfonso, B., \& Nieves, Z. I. (2013). Adaptación del inventario SISCO del estrés académico en estudiantes universitarios cubanos de Ciencias Médicas (Tesis de maestría, Universidad Central Marta Abreu de Las Villas). https://dspace.uclv.edu.cu/bitstream/ handle/123456789/3078/Tesis\%20Paginada\%20Belkis\%20alfonso\%20Aguila.pdf? sequence $=1 \&$ is Allowed $=\mathrm{y}$

Barraza, A. (2006). Un modelo conceptual para el estudio del estrés académico. Revista electrónica de psicología iztacala, 9(3). https://www.psicologiacientifica.com/estres-academico-modelo-conceptual/

Barraza, A. (2010). Validación del inventario de expectativas de autoeficacia académica en tres muestras secuenciales e independientes. CPU-e, Revista de Investigación Educativa, (10), 1-30.https://www.uv.mx/cpue/num10/inves/completos/barraza_validacion.html

Barraza, A., \& Acosta, M. (2007). El estrés de examen en educación media superior CASO. Colegio de Ciencias y Humanidades de la Universidad Juárez del Estado de Durango. Innovación Educativa, 7(37),16-37. http://www.redalyc.org/articulo. oa? id=179420820003

Berrío, N., \& Mazo, R. (2011). Estrés Académico. Revista de Psicología Universidad de Antioquia., 3(2). 
Caldera, J. F., Pulido, B. E., \& Martínez, G. (2007). Niveles de estrés y rendimiento académico en estudiantes de la carrera de Psicología del Centro Universitario de Los Altos. Revista de Educación y Desarrollo, 7, 77-82. http://www.cucs.udg.mx/ revistas/edu_desarrollo/anteriores/7/007_Caldera.pdf

Díaz Martín, Y. (2010). Estrés académico y afrontamiento en estudiantes de Medicina. Humanidades Médicas, 10(1). http://scielo.sld.cu/scielo.php?pi$\mathrm{d}=\mathrm{S} 1727-81202010000100007 \&$ script $=$ sci_arttext

Fernández, L. (2007). Pensando en la personalidad. Tomo 2. Editorial Félix Varela.Polo, A., Hernández López, J. M., \& Pozo Muñoz, C. (1996). Evaluación del estrés académico en estudiantes universitarios. Ansiedad y estrés, 2(2), 159-172.

Zaldívar, D. (2011). Psicología, salud y bienestar. Editorial. Liber, Instituto Cubano del Libro. 